\title{
Dipeptidyl peptidase-4 inhibitor decreases the risk of atrial fibrillation in patients with type 2 diabetes: a nationwide cohort study in Taiwan
}

\author{
Chia-Yu Chang ${ }^{1 \dagger}$, Yung-Hsin Yeh ${ }^{1,2+}$ (D), Yi-Hsin Chan ${ }^{1,2,3}$, Jia-Rou Liu ${ }^{4,5}$, Shang-Hung Chang ${ }^{1,2}$, Hsin-Fu Lee ${ }^{1,2}$, \\ Lung-Sheng $\mathrm{Wu}^{1,2}$, Kun-Chi Yen ${ }^{1,2}$, Chi-Tai Kuo ${ }^{1,2}$ and Lai-Chu See ${ }^{4,5,6^{*}}$
}

\begin{abstract}
Background: Whether dipeptidyl peptidase-4 inhibitor (DPP4i) is associated with a lower risk of new-onset atrial fibrillation (AF) in patients with diabetes remains unclear. This study aimed to evaluate the risk of AF associated with use of DPP4i among a longitudinal cohort of patients with diabetes.

Methods: Over a 3-year period, 480,000 patients with diabetes were analyzed utilizing Taiwan's National Health Insurance Research Database and 90,880 patients taking metformin as first-line therapy were enrolled. Patients were further divided into two groups: (1) DPP4i users: those taking DPP4i and (2) non-DPP4i users: those prescribed other hypoglycemic agents (HAs) as second-line drug. Study end point was defined by diagnosis of AF, addition of any third-line HA, or the end of the study period (December 31, 2013), whichever came first.

Results: A total of 16,017 DPP4i users and 74,863 non-DPP4i users were eligible for the study. For the DPP4i group, most patients were prescribed sitagliptin $(n=12,180 ; 76 \%)$. Among the non-DPP4i group, most patients took sulfonylurea ( $n=60,606 ; 81 \%)$ as their second-line medication. DPP4i users were associated with a lower risk of new-onset AF compared with non-DPP4i users after propensity-score weighting (hazard ratio $0.65 ; P<0.0001$ ). Subgroup analysis showed that DPP4i user were associated with a lower risk of new-onset AF compared with non-DPP4i users in most subgroups. Multivariate analysis indicated that use of DPP4i was associated with lower risk of new-onset AF and age > 65 years, presence of hypertension, and ischemic heart disease were independent risk factors for new-onset AF.
\end{abstract}

Conclusions: Among patients with diabetes prescribed with metformin, the patients with DPP4i as second HA were associated with a lower risk of AF compared with the patients with other drugs as second HAs in real-world practice.

Keywords: Dipeptidyl peptidase-4 inhibitor, Type 2 diabetes mellitus, Atrial fibrillation

\section{Introduction}

Atrial fibrillation (AF) is the most common cardiac arrhythmia and significantly increases the risk of comorbidity and mortality $[1,2]$ with a threefold increased risk of heart failure and a fivefold increased risk of stroke

\footnotetext{
*Correspondence: lichu@mail.cgu.edu.tw

${ }^{\dagger}$ Chia-Yu Chang and Yung-Hsin Yeh contributed equally to the study and manuscript

${ }^{4}$ Department of Public Health, College of Medicine, Chang Gung University, No. 259, Wenhua 1st Rd., Guishan, Taoyuan 33302, Taiwan Full list of author information is available at the end of the article
}

[3-6]. As the world population ages, the prevalence of AF is predicted to increase by 2.5 fold in the next 50 years [7]. Diabetes mellitus (DM) is an important independent risk factor for $\mathrm{AF}$ [8-12]. In a previous study, AF occurred in $14.9 \%$ of diabetic patients and $10.3 \%$ in nondiabetic patients [8]. Furthermore, diabetes was highly associated with the prevalence of metabolic syndrome, which is also associated with a higher risk for AF [8]. Alogliptin, a dipeptidyl peptidase-4 inhibitor (DPP-4i), not only has anti-hyperglycemic effects, but can also inhibit the maintenance of AF induced by tachy-pacing, 
as shown in a recent animal study [13]. However, Only a few studies investigated if DPP4 $\mathrm{i}$ has cardiac protective effects including AF [14-16]. This study had as its underlying hypothesis that DPP4i could potentially reduce the incidence of AF in type- 2 diabetic patients. The goal of the present study was to evaluate the risk of AF associated with use of DPP4i in a nationwide cohort study of diabetic patients in Taiwan.

\section{Materials and methods}

\section{Data source}

This study was approved by the Institutional Review Board of Chang Gung Memorial Hospital, Linkou, Taiwan. Informed consent was waived because the original identification number of each patient in the National Health Insurance (NHI) research database (NHIRD) of Taiwan was encrypted and de-identified to protect their privacy. The NHI program is a compulsory universal health insurance program in Taiwan which provides comprehensive medical care coverage to more than $99 \%$ of Taiwanese residents. The NHIRD of the National Health Research Institutes of Taiwan included detailed health care information for 23.72 million enrollees in 2014 [17].

\section{Study cohort and outcomes}

From 2009 to 2012, 480,000 patients with diabetes were analyzed utilizing a Longitudinal Cohort of Diabetes Patients Database (LHDB) using newly diagnosed DM codes based on the International Classification of Diseases, ninth revision, Clinical Modification (ICD-9-CM) as previous described [18]. A flowchart of enrollment of the study cohort is summarized in Fig. 1. Subjects who were less than 20 years of age $(n=5526)$, had the diagnosis of AF $(n=10,388)$, or any cardiovascular event $(n=27,834)$ before the diagnosis of diabetes were excluded from the study. In Taiwan, metformin is considered a first-line hypoglycemic agent (HA) according to the current guidelines. Second-line HAs (which include sulfonylurea, alpha glucosidase inhibitor, thiazolidinedione (TZD), meglitinide, insulin, GLP-1 analogue, and DPP4i) are administered when inadequate therapeutic response to metformin is suspected, according to the current guidelines and payment criteria of the NHI in Taiwan. Subjects who were not prescribed any HA during their whole treatment course, who were first prescribed a HA other than metformin, who were only prescribed metformin during their whole treatment course, or who were prescribed with any HAs before the diagnosis of diabetes were also excluded from the current study ( $\mathrm{n}=341,457)$. Finally, 90,880 patients with diabetes taking metformin as the first-line therapy were enrolled in the study. Study subjects were further divided into two groups: those taking DPP4i (the DPP4i group) versus those prescribed other HAs as second-line drug (the non-DPP4i group). The DPP4i group $(n=16,017)$ was defined by the use of DPP4i as the second-line HA. The non-DPP4i group $(n=74,863)$ was defined by the use of other HAs as the second-line HA. The first claim date of the DPP4i group or the nonDPP4i group was defined as the drug index date. The study outcome was defined by the diagnosis of AF based on the ICD-9-CM code of 427.31, in either an in-patient or outpatient department at least once. The follow-up period was defined from the index date until the occurrence of the first study outcome (AF), the addition of any new HA due to inadequate sugar control, or the end of the study period (December 31, 2013), whichever came first.

\section{Covariates}

Risk factors for cardiovascular events and use of medication at baseline were obtained from claim records using the above diagnoses or medication codes prior to the index date. A history of specific prescribed medicines was confined to medications used at least once within the 3 months preceding the index date. The ICD-9-CM codes used to identify the study outcomes and covariates are summarized in Additional file 1: Table S1.

\section{Statistical analysis}

Propensity score method, which simulates the goldstandard of a randomized clinical trial (RCT) for observational data, was used to compare the effect between the two study groups on study outcomes. Inverse probability of treatment weighting (IPTW) of propensity scores was used to balance covariates across the two study groups [19]. The balance of potential confounders at baseline (index date) between the two study groups was evaluated by using standardized mean difference (SMD), rather than using statistical testing, because balance is a property of the sample and not of an underlying population. The value of absolute of SMD $\leq 0.1$ indicates a negligible difference in potential confounders between the two study groups. Risk of study outcomes over time for the DPP-4 inhibitor group compared with non-DPP-4 inhibitor group (reference) was obtained by using survival analysis (Kaplan-Meier method for univariate analysis and Cox proportional hazards regression for multivariate analysis) after IPTW. Subgroup analysis was performed to determine whether the DPP4i group continued to have a lower risk of new-onset AF when compared with non-DPP4i in subgroups. Statistical significance was defined at a $P$ value $<0.05$. All statistical analyses were performed using SAS 9.3 (SAS Institute Inc., Cary, North Carolina). 


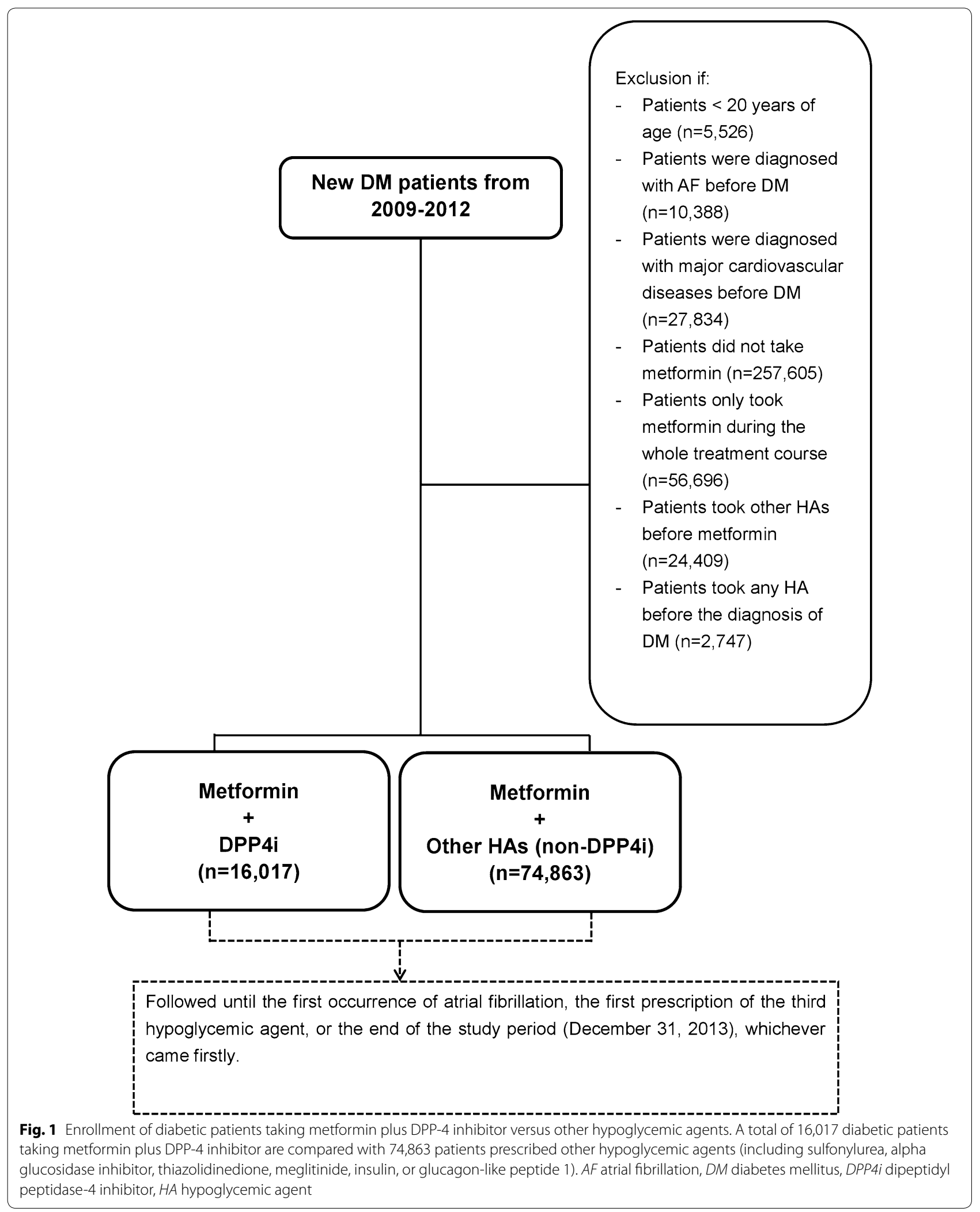




\section{Results}

A total of 16,017 DPP4i users and 74,863 non-DPP4i users were eligible for the study. Most patients in the DPP4i group were prescribed sitagliptin $(n=12,180$, $76 \%$ ); while 291, 1501 and 2045 patients were prescribed linagliptin (2\%), saxagliptin (9\%), and vildagliptin (13\%), respectively. Among the non-DPP4i group, most patients were prescribed sulfonylurea $(\mathrm{n}=60,606,81 \%)$ as the second-line HA. In addition, 4087, 4783, 2334, 1032, and five patients were prescribed alpha glucosidase inhibitor (5\%), meglitinide (6\%), thiazolidinedione (3\%), insulin (1\%), and GLP-1 analogue (0\%), respectively. There were 2016 patients (4\%) taking more than two second-line HAs concurrently.

Table 1 summarizes the baseline demographic characteristics, comorbidities, and medication differences between the two groups. Before propensity score weighting, the DPP4i group had a higher use of statins and angiotensin-converting enzyme inhibitor/angiotensin receptor blockers than non-DPP4i group, while age, gender, comorbidities and other medications were all similar between two study groups at baseline (all ASMD < 0.1). After propensity-score weighting, the two study groups were well-balanced in all characteristics (all ASMD $<0.1)$.

DPP4i users were associated with a lower risk of new-onset AF compared with non-DPP4i users, either before or after propensity-score weighting [hazard ratio (HR): 0.65 ; 95\% confidential interval (CI) $0.56-$ 0.76; $P<0.0001]$. It was noted that most HAs, with the exception of insulin/GLP1, were associated with a significantly higher risk of new-onset AF when compared with DPP4i (Table 2). Figure 2 and Additional file 2: Figure S1 show a clear separation of event curves for

Table 1 Baseline characteristics of diabetic patients taking metformin plus DPP4i versus other hypoglycemic agents, before and after propensity score weighting

\begin{tabular}{|c|c|c|c|c|c|c|}
\hline & \multicolumn{3}{|c|}{ Before weighting } & \multicolumn{3}{|c|}{ After weighting } \\
\hline & $\begin{array}{l}\text { DPP4i users } \\
(n=16,017)\end{array}$ & $\begin{array}{l}\text { Non-DPP4i users } \\
(n=74,863)\end{array}$ & $\begin{array}{l}\text { Standardized mean } \\
\text { difference }\end{array}$ & $\begin{array}{l}\text { DPP4i users } \\
(n=16,017)\end{array}$ & $\begin{array}{l}\text { Non-DPP4i users } \\
(n=74,863)\end{array}$ & $\begin{array}{l}\text { Standardized mean } \\
\text { difference }\end{array}$ \\
\hline \multicolumn{7}{|l|}{ Follow-up time (years) } \\
\hline Mean \pm SD & $2.04 \pm 1.21$ & $2.41 \pm 1.27$ & & $2.07 \pm 2.90$ & $2.41 \pm 1.39$ & \\
\hline \multicolumn{7}{|l|}{ Age at index date } \\
\hline Mean \pm SD & $54.51 \pm 12.53$ & $54.88 \pm 12.20$ & & $54.43 \pm 30.16$ & $54.88 \pm 13.41$ & \\
\hline$<65$ year & $80.23 \%$ & $79.59 \%$ & 0.0162 & $79.87 \%$ & $79.70 \%$ & 0.0043 \\
\hline$\geq 65$ years & $19.77 \%$ & $20.41 \%$ & & $20.13 \%$ & $20.30 \%$ & \\
\hline Gender & & & 0.0162 & & & -0.0008 \\
\hline Female & $42.64 \%$ & $41.84 \%$ & & $41.95 \%$ & $41.99 \%$ & \\
\hline \multicolumn{7}{|l|}{ History of comorbidity } \\
\hline Hypertension & $58.33 \%$ & $57.34 \%$ & 0.0199 & $57.38 \%$ & $57.52 \%$ & -0.0029 \\
\hline Hyperlipidemia & $59.76 \%$ & $56.96 \%$ & 0.0567 & $57.51 \%$ & $57.44 \%$ & 0.0014 \\
\hline Ischemic heart disease & $2.57 \%$ & $2.02 \%$ & 0.0386 & $2.10 \%$ & $2.11 \%$ & -0.0006 \\
\hline Heart valve surgery & $0.09 \%$ & $0.05 \%$ & 0.0200 & $0.05 \%$ & $0.05 \%$ & -0.0032 \\
\hline $\begin{array}{l}\text { Obstructive sleep } \\
\text { apnea }\end{array}$ & $0.00 \%$ & $0.00 \%$ & & $0.00 \%$ & $0.00 \%$ & \\
\hline Hyperthyroidism & $2.68 \%$ & $2.08 \%$ & 0.0406 & $2.19 \%$ & $2.19 \%$ & 0.0003 \\
\hline Chronic kidney disease & $7.56 \%$ & $7.09 \%$ & 0.0182 & $7.11 \%$ & $7.17 \%$ & -0.0024 \\
\hline PAOD & $0.51 \%$ & $0.36 \%$ & 0.0239 & $0.36 \%$ & $0.39 \%$ & -0.0038 \\
\hline Gout & $19.31 \%$ & $20.61 \%$ & -0.0323 & $20.37 \%$ & $20.39 \%$ & -0.0006 \\
\hline Chronic lung disease & $1.29 \%$ & $1.35 \%$ & -0.0049 & $1.31 \%$ & $1.34 \%$ & -0.0026 \\
\hline Congestive heart failure & $0.28 \%$ & $0.13 \%$ & 0.0355 & $0.15 \%$ & $0.16 \%$ & -0.0015 \\
\hline \multicolumn{7}{|c|}{ Medication } \\
\hline Beta-blocker & $13.78 \%$ & $13.75 \%$ & 0.0008 & $13.67 \%$ & $13.75 \%$ & -0.0026 \\
\hline Diltiazem/verapamil & $3.02 \%$ & $2.38 \%$ & 0.0408 & $2.48 \%$ & $2.49 \%$ & -0.0002 \\
\hline Statin & $33.60 \%$ & $25.83 \%$ & 0.1745 & $27.18 \%$ & $27.20 \%$ & -0.0005 \\
\hline ACEI/ARB & $36.62 \%$ & $30.55 \%$ & 0.1305 & $31.66 \%$ & $31.61 \%$ & 0.0012 \\
\hline
\end{tabular}

$A C E l$ angiotensin-converting-enzyme inhibitor, $A F$ atrial fibrillation, $A R B$ angiotensin II receptor antagonists, $C l$ confidence interval, $D M$ diabetes mellitus, $D P P 4 i$ dipeptidyl peptidase-4 inhibitor, GLP-1 glucagon-like peptide-1, $P A O D$ peripheral arterial obstructive disease, $T Z D$ thiazolidinedione 
Table 2 Incidence (per 100 person-years) of new-onset AF in diabetic patients taking metformin plus DPP4i or other hypoglycemic agents

\begin{tabular}{|c|c|c|c|c|c|}
\hline & \multicolumn{5}{|c|}{ New-onset AF } \\
\hline & Numbers & Events & Incidence before weighting & Incidence after weighting & $\begin{array}{l}\mathrm{HR}^{\mathrm{a}} \\
95 \% \mathrm{Cl} \\
\text { P value }\end{array}$ \\
\hline DPP4i & 16,017 & 45 & $0.14(0.10-0.18)$ & $0.14(0.12-0.15)^{*}$ & $\begin{array}{l}1.00 \\
\text { (Reference) }\end{array}$ \\
\hline Other hypoglycemic agents rather than DPP4i & 74,863 & 386 & $0.21(0.19-0.24)$ & $0.21(0.19-0.23)$ & $\begin{array}{l}1.53 \\
(1.31-1.78) \\
P<0.0001\end{array}$ \\
\hline Sulfonylurea & 62,216 & 318 & $0.20(0.18-0.23)$ & $0.20(0.18-0.23)$ & $\begin{array}{l}1.45 \\
(1.24-1.70) \\
P<0.0001\end{array}$ \\
\hline Alpha glucosidase inhibitor & 5091 & 24 & $0.24(0.14-0.33)$ & $0.23(0.15-0.32)$ & $\begin{array}{l}1.75 \\
(1.19-2.57) \\
P=0.0045\end{array}$ \\
\hline Meglitinide & 5164 & 41 & $0.38(0.26-0.49)$ & $0.38(0.27-0.48)$ & $\begin{array}{l}2.78 \\
(2.05-3.76) \\
P<0.0001\end{array}$ \\
\hline TZD & 3091 & 15 & $0.23(0.11-0.34)$ & $0.23(0.12-0.33)$ & $\begin{array}{l}1.68 \\
(1.05-2.69) \\
P=0.0307\end{array}$ \\
\hline Insulin & 1361 & 3 & $0.11(0.02-0.32)$ & $0.11(0.03-0.29)$ & $\begin{array}{l}0.79 \\
(0.28-2.26) \\
P=0.20\end{array}$ \\
\hline GLP1 & 5 & 0 & & & \\
\hline
\end{tabular}

There were 2016 patients taking more than two hypoglycemic agents as second-line hypoglycemic agents at the same time

$A C E l$ angiotensin-converting-enzyme inhibitor, $A F$ atrial fibrillation, $A R B$ angiotensin II receptor antagonists, $C l$ confidence interval, $D M$ diabetes mellitus, $D P P 4 i$ dipeptidyl peptidase-4 inhibitor, GLP-1 glucagon-like peptide-1; $P A O D$ peripheral arterial obstructive disease, TZD thiazolidinedione

${ }^{a}$ For other hypoglycemic agents versus DPP-4 inhibitors (reference) after propensity score weighting

new-onset AF between these two groups either before or after propensity score weighting adjustment. The time interval from the index day to the occurrence of AF in DPP4i user vs non-user is $1.9 \pm 2.9$ years and $1.7 \pm 1.2$ years respectively. Some patients did not take DPP4i at the diagnosis of the first AF. Among the $45 \mathrm{AF}$ events in DPP4i users, 10 of them (22.2\%) did not take DPP4i within 3 months of the events. Subgroup analysis revealed that DPP4i usage was associated with a lower risk of new-onset AF compared with non-DPP4i usage in most subgroups (Fig. 3).

In Table 3, Cox's model was performed after propensity score weighting in order to identify the independent risk factors for the new-onset AF for those patients taking HAs. The multivariate analysis indicated that use of DPP4i was associated with lower risk of newonset AF (HR 0.69; 95\% CI 0.59-0.81; $P<0.0001$ ), and age $>65$ years (HR 4.75; 95\% CI 4.07-5.55; $P<0.0001)$, presence of hypertension (HR 1.74; 95\% CI 1.45-2.06; $P<0.0001$ ), and ischemic heart disease (HR 1.98; 95\% CI $1.48-2.66 ; P<0.0001)$ were independent risk factors for new-onset AF.

\section{Discussion}

The nationwide cohort study evaluated the risk of newonset $\mathrm{AF}$ in metformin-based patients with diabetes taking DPP4i versus other second-line hypoglycemic agents. Recently we had presented metformin users were associated with a lower risk of AF in patients with diabetes compared with non-users [20, 21]. In this study, in addition to use of metformin, we observed that patients taking DPP4i had a significantly lower risk of new-onset AF than those treated with other HAs including sulfonylurea, alphaglucosidase inhibitors, meglitinide, and thiazolidinedione. The risk reduction of new-onset AF for DPP4i users versus other HAs was similar among most subgroups.

Several studies have indicated that diabetes, as well as age, hypertension, and structural heart diseases, are independent risk factors for AF [22]. Atrial fibrosis and chronic inflammation are known to contribute to AF $[23,24]$. Diabetes is associated with numerous metabolic defects which could be responsible for AF occurrence. Diabetes could also cause structural, electrical, electromechanical and autonomic remodeling, triggering $\mathrm{AF}$ in patients with diabetes [25]. In several animal studies, 


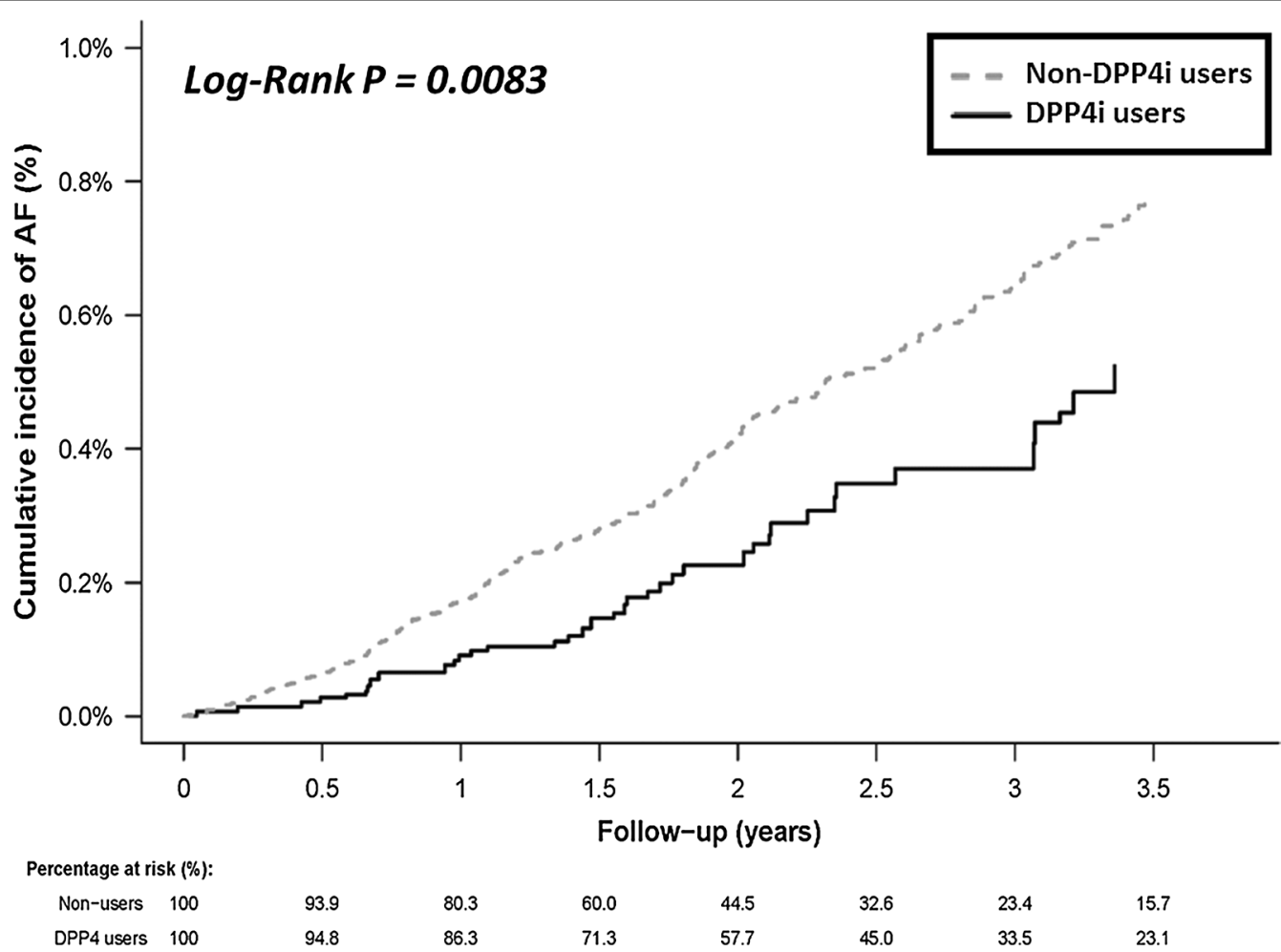

Fig. 2 Cumulative risk curve of new-onset AF for the study cohorts treated with metformin plus DPP-4 inhibitor versus other hypoglycemic agents after propensity score weighting. DPP4i group (solid line) shows a significantly lower cumulative risk of new-onset AF compared with non-DPP4i group in patients treated with metformin (dotted line). DPP4i dipeptidyl peptidase-4 inhibitor

DPP4i inhibitors increase in threshold of ventricular fibrillation during the ischemic period and stabilized cardiac electrophysiology, protected cardiac mitochondrial function [26]. DPP4i is commonly used for the treatment of patients with diabetes in clinical practice. By inhibiting the degradation of GLP-1, DPP4i has been shown to increase the serum levels of GLP-1, which indirectly stimulate insulin secretion and enhance beta-cell function. DPP-4 is highly expressed in endothelial cells and the GLP-1 receptor is expressed on cardiomyocytes, vascular smooth muscle cells, and endothelial cells. A previous animal study showed that DPP4i had both GLP-1-dependent and GLP-1-independent cardioprotective effects using an ischemic heart model [27]. A recent study also showed that DPP4i may exert antiarrhythmic effects and reduce infarct size during myocardial ischemia and reperfusion [28]. There were several mechanisms to explain the relationship between diabetes and AF and the mechanism of DPP4i in lowering AF risk. Recently Chang et al. showed that in spontaneously hypertensive rats, sitagliptin would modulate the electrical and mechanical properties of pulmonary veins and atria, suggesting that DPP4i may be protective against AF genesis [29]. Furthermore, Yamamoto et al. [13]. demonstrated that alogliptin, a
DPP4i, can shorten the AF duration caused by ventricular tachy-pacing in rabbits with fibrotic atria. The underlying mechanisms may include augmentation of atrial remodeling and improvement of mitochondrial function [30]. Further, administration of nitric oxide synthase inhibitor has been shown to block the protective effects of alogliptin via shortening AF duration, capillary density, and atrial fibrosis. Their findings suggest that DPP4i may have an antiarrhythmic effect in the prevention of heartfailure-induced AF [13]. In contrast, Hayami et al. [31]. demonstrated that administration of sulfonylurea and DPP4i both inhibited inflammation and fibrosis of the atria in streptozotocin-induced diabetic rats. However, no significant differences were observed between the two oral HAs. They concluded that reduced atrial fibrosis may derive from the tight control of blood glucose levels rather than a drug-specific anti-inflammatory property.

The monotherapy of metformin is suggested as firstline therapy for glycemic control in newly diagnosed patients with type 2 diabetes according to the current guidelines [32]. If monotherapy does not achieve the therapeutic goal, a second HA would be added [33]. Several studies have indicated that patients with diabetes have an increased risk of developing adverse cardiovascular 


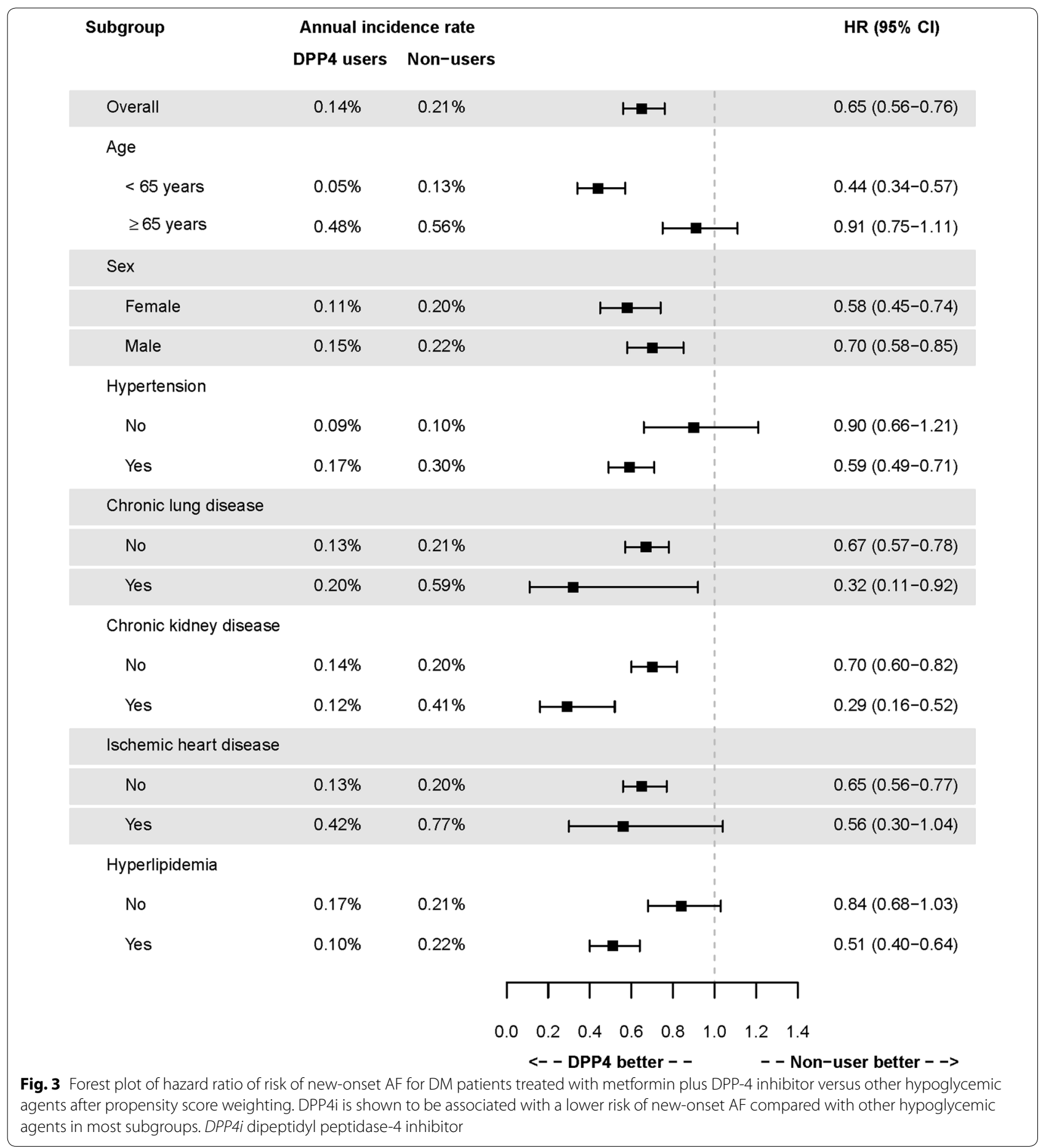

outcomes [34]. Therefore, prevention of any adverse cardiovascular outcome seems to be an important consideration when choosing second-line HAs. Until now, only empagliflozin, a potent inhibitor of sodium glucose cotransporter 2 (SGLT2), and metformin have provided cardioprotective effects in patients with diabetes beyond the hypoglycemic effects $[35,36]$. It is unclear if GLP-1 receptor agonists was associated with AF [37]. At the present time, it is still unclear whether DPP4i would lead to better outcomes with a reduction in incidence of major adverse cardiovascular events [38-41]. Recent large scale clinical trials, including EXAMINE, 
Table 3 Predictors of new-onset AF for diabetic patients taking hypoglycemic agents after propensity score weighting

\begin{tabular}{|c|c|c|}
\hline & \multicolumn{2}{|c|}{ Hazard ratio $(95 \% \mathrm{Cl}) ; \mathrm{P}$ value } \\
\hline & Univariate & Multivariate \\
\hline DPP4i versus other hypoglycemic agents & $0.65(0.56-0.76) ;<0.0001$ & $0.69(0.59-0.81) ; P<0.0001$ \\
\hline \multicolumn{3}{|l|}{ Age (years) } \\
\hline$<65$ & 1.00 (reference) & 1.00 (reference) \\
\hline$\geq 65$ & $5.76(4.97-6.68) ;<0.0001$ & $4.75(4.07-5.55) ; P<0.0001$ \\
\hline Female gender & $0.87(0.75-1.01) ; 0.0714$ & \\
\hline Chronic lung disease & $2.28(1.46-3.56) ; 0.0003$ & \\
\hline Chronic kidney disease & $1.74(1.37-2.21) ;<0.0001$ & \\
\hline Hypertension & $2.64(2.22-3.13) ;<0.0001$ & $1.74(1.45-2.06) ; P<0.0001$ \\
\hline Ischemic heart disease & $3.84(2.88-5.13) ;<0.0001$ & $1.98(1.48-2.66) ; P<0.0001$ \\
\hline
\end{tabular}

$A C E l$ angiotensin-converting-enzyme inhibitor, $A F$ atrial fibrillation, $A R B$ angiotensin II receptor antagonists, DM diabetes mellitus, DPP4i dipeptidyl peptidase-4 inhibitor, GLP-1 glucagon-like peptide-1, PAOD peripheral arterial obstructive disease, TZD thiazolidinedione

SAVOR-TIMI53, and TECOS, all failed to show significant improvement in cardiovascular outcomes in type 2 diabetic patients treated with DPP4i $[38,42,43]$. In contrast, several nationwide cohort studies presented that DPP4i may have cardioprotective properties, including the ability to lower the incidence of heart failure, coronary heart disease, and stroke [15, 39, 44]. In subgroup analysis (Fig. 3), however, we did not find differences in the occurrence of new-onset AF between DPP4 users and non-users in patients $>65$ years. It is likely that more AF-precipitating factors coexist in aged diabetic patients, which would remove the protective effect of DPP4i. Until now, no randomized control trials directly compared the risk of new-onset $\mathrm{AF}$ in patients with diabetes taking DPP4i compared with patients taking other HAs. Our data revealed that DPP4i was associated with a lower risk of new-onset $\mathrm{AF}$ in patients with diabetes, indicating that DPP4 $i$ as a feasible second-line oral HA for AF primary prevention. Future prospective studies are necessary, however, to evaluate the potential role of DPP4i on atrial remodeling and AF prevention in the setting of diabetes.

\section{Study limitations}

Our study had several limitations including lack of laboratory data such as hemoglobin A1c levels, blood sugar levels, renal function, and lipid profiles, thus, the severity of diabetes in each patient could not be classified. Furthermore, because of the lack of EKG data, the contribution of persistent AF or paroxysmal AF to acute illness could not be assessed. In addition, although an extensive number of variables had been selected for our propensity score model, and a close balance among those factors was successfully achieved in our study, there were other unmeasured confounding factors that may have biased our results including the use of tobacco and/or alcohol, body mass index, family history, and physicians' preference for a specific HA. Also, coding errors regarding outcomes and comorbidities may have existed because of each physician's different response when caring for their own patients. Finally, this was a retrospective, observational study. Therefore, further prospective randomized studies are needed to determine whether our findings are applicable to non-Asian patients with type 2 diabetes.

\section{Conclusions}

Among patients with diabetes prescribed with metformin, the patients with DPP4i as second HA were associated with a lower risk of AF compared with the patients with other drugs as second HAs in real-world practice.

\section{Additional files}

Additional file 1: Table S1. International Classification of Disease (9th edition) Clinical Modification (ICD 9-CM) codes used to define the comorbidities and clinical outcome in the study cohort.

Additional file 2: Figure S1. Cumulative risk curve of the new-onset AF for the study cohorts treated with metformin plus DDP-4 inhibitor or other hypoglycemic agents before propensity score weighting. DDP4i group (solid line) had a significantly lower cumulative risk of new-onset AF compared with non-DDP4i group in patients treated with metformin (dotted line). DPP4i dipeptidyl peptidase-4 inhibitor.

\section{Abbreviations}

AF: atrial fibrillation; ASMD: absolute standardized mean difference; DM: diabetes mellitus; DPP4i: dipeptidyl peptidase-4 inhibitor; HA: hypoglycemic agents; ICD-9-CM: International Classification of Diseases, ninth revision, Clinical Modification; IPTW: inverse probability of treatment weighting; RCT: randomized clinical trial; LHDB: Longitudinal Cohort of Diabetes Patients Database; NHIRD: National Health Insurance Research Database.

\section{Authors' contributions}

Conceived and designed the experiments: CYC, YHY, YHC, LCS. Performed the experiments: JRL, SHC, CTK. Analyzed the data: JRL, LCS. Contributed reagents/ materials/analysis tools: HFL, LSW. Wrote the manuscript: CYC, YHY, YHC. Overall responsibility: YHY and LCS. All authors read and approved the final manuscript. 


\begin{abstract}
Author details
${ }^{1}$ College of Medicine, Chang Gung University, Taoyuan 33302, Taiwan.

2 Department of Cardiology, Chang Gung Memorial Hospital, Linkou,

Taoyuan 33305, Taiwan. ${ }^{3}$ Microscopy Core Laboratory, Chang Gung Memorial Hospital, Linkou, Taoyuan 33305, Taiwan. ${ }^{4}$ Department of Public Health, College of Medicine, Chang Gung University, No. 259, Wenhua 1st Rd., Guishan, Taoyuan 33302, Taiwan. ${ }^{5}$ Biostatistics Core Laboratory, Molecular Medicine Research Center, Chang Gung University, Taoyuan 33302, Taiwan. ${ }^{6}$ Division of Rheumatology, Allergy and Immunology, Chang Gung Memorial Hospital, Linkou, Taoyuan 33305, Taiwan.
\end{abstract}

\section{Acknowledgements}

None.

\section{Competing interests}

The authors declare that they have no competing interests.

\section{Availability of data and materials}

The NHI program is a compulsory universal health insurance program in Taiwan which provides comprehensive medical care coverage to more than $99 \%$ of Taiwanese residents. The NHIRD of the National Health Research Institutes of Taiwan included detailed health care information for 23.72 million enrollees in 2014 [17].

\section{Consent for publication}

All authors consented for the publication of the manuscript.

\section{Ethics approval and consent to participate}

This study was approved by the Institutional Review Board of Chang Gung Memorial Hospital, Linkou, Taiwan. Informed consent was waived because the original identification number of each patient in the National Health Insurance $(\mathrm{NHI})$ research database (NHIRD) of Taiwan was encrypted and de-identified to protect their privacy.

\section{Funding}

This study was supported by Grants 102-2628-B-182 -011-MY3,

102-2314-B-182A-053-MY3 from the Ministry of Science and Technology and CMRPG3F0991-3, CMRPG3D1371-3 and CMRPD1F0251 from the Chang Gung Memorial Hospital, Linkou, Taiwan.

\section{Publisher's Note}

Springer Nature remains neutral with regard to jurisdictional claims in published maps and institutional affiliations.

Received: 1 September 2017 Accepted: 6 December 2017

Published online: 19 December 2017

\section{References}

1. Piccini JP, Hammill BG, Sinner MF, Jensen PN, Hernandez AF, Heckbert $\mathrm{SR}$, et al. Incidence and prevalence of atrial fibrillation and associated mortality among Medicare beneficiaries, 1993-2007. Circ Cardiovasc Qual Outcomes. 2012;5(1):85-93.

2. Benjamin EJ, Wolf PA, D'Agostino RB, Silbershatz H, Kannel WB, Levy D. Impact of atrial fibrillation on the risk of death: the Framingham Heart Study. Circulation. 1998;98(10):946-52.

3. Lin HJ, Wolf PA, Kelly-Hayes M, Beiser AS, Kase CS, Benjamin EJ, et al. Stroke severity in atrial fibrillation. The Framingham Study. Stroke. 1996;27(10):1760-4

4. Patel NJ, Deshmukh A, Pant S, Singh V, Patel N, Arora S, et al. Contemporary trends of hospitalization for atrial fibrillation in the United States, 2000 through 2010: implications for healthcare planning. Circulation. 2014;129(23):2371-9.

5. Wang TJ, Larson MG, Levy D, Vasan RS, Leip EP, Wolf PA, et al. Temporal relations of atrial fibrillation and congestive heart failure and their joint influence on mortality: the Framingham Heart Study. Circulation. 2003;107(23):2920-5.
6. Chang SH, Kuo CF, Chou IJ, See LC, Yu KH, Luo SF, Huang LH, Zhang W, Doherty M, Wen MS, Kuo CT, Yeh YH. Association of a family history of atrial fibrillation with incidence and outcomes of atrial fibrillation: a population-based family cohort study. JAMA Cardiol. 2017;2(8):863-70.

7. Go AS, Hylek EM, Phillips KA, Chang Y, Henault LE, Selby JV, et al. Prevalence of diagnosed atrial fibrillation in adults: national implications for rhythm management and stroke prevention: the AnTicoagulation and Risk Factors in Atrial Fibrillation (ATRIA) Study. JAMA. 2001;285(18):2370-5.

8. Movahed MR, Hashemzadeh M, Jamal MM. Diabetes mellitus is a strong, independent risk for atrial fibrillation and flutter in addition to other cardiovascular disease. Int J Cardiol. 2005;105(3):315-8.

9. De Sensi F, De Potter T, Cresti A, Severi S, Breithardt G. Atrial fibrillation in patients with diabetes: molecular mechanisms and therapeutic perspectives. Cardiovasc Diagn Ther. 2015;5(5):364-73.

10. Huxley RR, Filion KB, Konety S, Alonso A. Meta-analysis of cohort and case-control studies of type 2 diabetes mellitus and risk of atrial fibrillation. Am J Cardiol. 2011;108(1):56-62.

11. Plitt A, McGuire DK, Giugliano RP. Atrial fibrillation, type 2 diabetes, and non-vitamin k antagonist oral anticoagulants: a review. JAMA Cardiol. 2017:2(4):442-8.

12. Grundvold I, Bodegard J, Nilsson PM, Svennblad B, Johansson G, Östgren CJ, et al. Body weight and risk of atrial fibrillation in 7,169 patients with newly diagnosed type 2 diabetes; an observational study. Cardiovasc Diabetol. 2015;14:5

13. Yamamoto T, Shimano M, Inden Y, Takefuji M, Yanagisawa S, Yoshida N, et al. Alogliptin, a dipeptidyl peptidase-4 inhibitor, regulates the atrial arrhythmogenic substrate in rabbits. Heart Rhythm. 2015;12(6):1362-9.

14. Persson F, Nyström T, Jørgensen ME, Carstensen B, Gulseth HL, Thuresson $M$, et al. Dapagliflozin is associated with lower risk of cardiovascular events and all-cause mortality in people with type 2 diabetes (CVD-REAL Nordic) when compared with dipeptidyl peptidase-4 inhibitor therapy: a multinational observational study. Diabetes Obes Metab; 2017. (Epub ahead of print)

15. Chen HY, Yang FY, Jong GP. Liou YS Antihyperglycemic drugs use and new-onset atrial fibrillation in elderly patients. Eur J Clin Invest. 2017;47(5):388-93.

16. Nakamura T, Iwanaga Y, Miyaji Y, Nohara R, Ishimura T, Miyazaki S. Cardiovascular efficacy of sitagliptin in patients with diabetes at high risk of cardiovascular disease: a 12-month follow-up. Cardiovasc Diabetol. 2016;15:54

17. National Health Insurance Administration, Ministry of Health and Welfare, Taiwan. The National Health Insurance Statistics; 2014. http://www.nhi.gov.tw/English/webdata/webdata. aspx?menu=11\&menu_id=296\&WD_ID=296\&webdata_id $=4835$.

18. Wu LS, Chang SH, Chang GJ, Liu JR, Chan YH, Lee HF, Wen MS, Chen WJ, Yeh YH, Kuo CT, See LC. A comparison between angiotensin converting enzyme inhibitors and angiotensin receptor blockers on end stage renal disease and major adverse cardiovascular events in diabetic patients: a population-based dynamic cohort study in Taiwan. Cardiovasc Diabetol. 2016;15(1):56.

19. Austin PC. The use of propensity score methods with survival or time-toevent outcomes: reporting measures of effect similar to those used in randomized experiments. Stat Med. 2014;33(7):1242-58.

20. Chang SH, Wu LS, Chiou MJ, Liu JR, Yu KH, Kuo CF, Wen MS, Chen WJ, Yeh YH, See LC. Association of metformin with lower atrial fibrillation risk among patients with type 2 diabetes mellitus: a population-based dynamic cohort and in vitro studies. Cardiovasc Diabetol. 2014;13(1):123.

21. Lee KT, Yeh YH, Chang SH, See LC, Lee CH, Wu LS, Liu JR, Kuo CT, Wen MS. Metformin is associated with fewer major adverse cardiac events among patients with a new diagnosis of type 2 diabetes mellitus: a propensity score-matched nationwide study. Medicine (Baltimore). 2017:96(28):e7507.

22. Benjamin EJ, Levy D, Vaziri SM, D'Agostino RB, Belanger AJ, Wolf PA. Independent risk factors for atrial fibrillation in a population-based cohort. The Framingham Heart Study. JAMA. 1994;271(11):840-4.

23. Ferrari R, Bertini M, Blomstrom-Lundqvist C, Dobrev D, Kirchhof P, Pappone C, Ravens U, Tamargo J, Tavazzi L, Vicedomini GG. An update on atrial fibrillation in 2014: from pathophysiology to treatment. Int J Cardiol. 2016;203:22-9. 
24. Aviles RJ, Martin DO, Apperson-Hansen C, Houghtaling PL, Rautaharju P, Kronmal RA, et al. Inflammation as a risk factor for atrial fibrillation. Circulation. 2003;108(24):3006-10.

25. Tadic M, Cuspidi C. Type 2 diabetes mellitus and atrial fibrillation: from mechanisms to clinical practice. Arch Cardiovasc Dis. 2015;108(4):269-76.

26. Wang MT, Lin SC, Tang PL, Hung WT, Cheng CC, Yang JS, et al. The impact of DPP4i inhibitors on long-term survival among diabetic patients after first acute myocardial infarction. Cardiovasc Diabetol. 2017;16(1):89.

27. Shah Z, Pineda C, Kampfrath T, Maiseyeu A, Ying Z, Racoma I, et al. Acute DPP-4 inhibition modulates vascular tone through GLP-1 independent pathways. Vasc Pharmacol. 2011:55(1-3):2-9.

28. Ihara M, Asanuma H, Yamazaki S, Kato H, Asano Y, Shinozaki Y, et al. An interaction between glucagon-like peptide-1 and adenosine contributes to cardioprotection of a dipeptidyl peptidase 4 inhibitor from myocardial ischemia-reperfusion injury. Am J Physiol Heart Circ Physiol. 2015;308(10):H1287-97

29. Chang CJ, Yang TF, Lee TI, Chen YC, Kao YH, Chen SA, et al. Sitagliptin modulates the electrical and mechanical characteristics of pulmonary vein and atrium. Acta Cardiol Sin. 2014;30(1):29-37.

30. Zhang $X$, Zhang Z, Zhao Y, Jiang N, Qiu J, Yang Y, et al. Alogliptin, a dipeptidyl peptidase-4 inhibitor, alleviates atrial remodeling and improves mitochondrial function and biogenesis in diabetic rabbits. J Am Heart Assoc. 2017;6(5):e005945

31. Hayami N, Sekiguchi A, Iwasaki YK, Murakawa Y, Yamashita T. No additional effect of DPP-4 inhibitor on preventing atrial fibrosis in streptozotocin-induced diabetic rat as compared with sulfonylurea. Int Heart J. 2016;57(3):336-40.

32. Palmer SC, Mavridis D, Nicolucci A, Johnson DW, Tonelli M, Craig JC, et al. Comparison of clinical outcomes and adverse events associated with glucose-lowering drugs in patients with type 2 diabetes: a meta-analysis. JAMA. 2016:316(3):313-24.

33. Bennett WL, Maruthur NM, Singh S, Segal JB, Wilson LM, et al. Comparative effectiveness and safety of medications for type 2 diabetes: an update including new drugs and 2-drug combinations. Ann Intern Med. 2011;154(9):602-13

34. Sarwar N, Gao P, Seshasai SR, Gobin R, Kaptoge S, Di Angelantonio E, et al. Diabetes mellitus, fasting blood glucose concentration, and risk of vascular disease: a collaborative meta-analysis of 102 prospective studies. Lancet. 2010;375(9733):2215-22.

35. Lamanna C, Monami M, Marchionni N, Mannucci E. Effect of metformin on cardiovascular events and mortality: a meta-analysis of randomized clinical trials. Diabetes Obes Metab. 2011;13(3):221-8.

36. Zinman B, Wanner C, Lachin JM, Fitchett D, Bluhmki E, Hantel S, et al. Empagliflozin, cardiovascular outcomes, and mortality in type 2 diabetes. N Engl J Med. 2015;373(22):2117-28

37. Monami M, Nreu B, Scatena A, Giannini S, Andreozzi F, Sesti G, et al. Glucagon-like peptide-1 receptor agonists and atrial fibrillation: a systematic review and meta-analysis of randomised controlled trials. J Endocrinol Invest. 2017;40(11):1251-8.

38. Scirica BM, Bhatt DL, Braunwald E, Steg PG, Davidson J, Hirshberg B, et al. Saxagliptin and cardiovascular outcomes in patients with type 2 diabetes mellitus. N Engl J Med. 2013;369(14):1317-26.

39. Yang TY, Liaw YP, Huang JY, Chang HR, Chang KW, Ueng KC. Association of Sitagliptin with cardiovascular outcome in diabetic patients: a nationwide cohort study. Acta Diabetol. 2016:53(3):461-8.

40. Zannad F, Cannon CP, Cushman WC, Bakris GL, Menon V, Perez AT, et al. Heart failure and mortality outcomes in patients with type 2 diabetes taking alogliptin versus placebo in EXAMINE: a multicentre, randomised, double-blind trial. Lancet. 2015:385(9982):2067-76.

41. Tomiyama H, Miwa T, Kan K, Matsuhisa M, Kamiya H, Nanasato M, et al. Impact of glycemic control with sitagliptin on the 2-year progression of arterial stiffness: a sub-analysis of the PROLOGUE study. Cardiovasc Diabetol. 2016;15(1):150.

42. White WB, Cannon CP, Heller SR, Nissen SE, Bergenstal RM, Bakris GL, et al. Alogliptin after acute coronary syndrome in patients with type 2 diabetes. N Engl J Med. 2013;369(14):1327-35.

43. Green JB, Bethel MA, Armstrong PW, Buse JB, Engel SS, Garg J, et al. Effect of sitagliptin on cardiovascular outcomes in type 2 diabetes. $\mathrm{N}$ Engl $J$ Med. 2015;373(3):232-42.

44. Ou HT, Chang KC, Li CY, Wu JS. Risks of cardiovascular diseases associated with dipeptidyl peptidase-4 inhibitors and other antidiabetic drugs in patients with type 2 diabetes: a nation-wide longitudinal study. Cardiovasc Diabetol. 2016:15:41.

\section{Submit your next manuscript to BioMed Central and we will help you at every step:}

- We accept pre-submission inquiries

- Our selector tool helps you to find the most relevant journal

- We provide round the clock customer support

- Convenient online submission

- Thorough peer review

- Inclusion in PubMed and all major indexing services

- Maximum visibility for your research

Submit your manuscript at www.biomedcentral.com/submit
O Biomed Central 